\title{
DETC2003/DAC-48812
}

\section{DESIGNING A DEXTEROUS RECONFIGURABLE PACKAGING SYSTEM FOR FLEXIBLE AUTOMATION}

\author{
Venketesh N Dubey \\ Bournemouth University \\ School of Design, Engineering \& Computing \\ 12 Christchurch Road, Studland House \\ Bournemouth, BH1 3NA, UK \\ Tel. +441202503791 \\ Fax. +44 1202503751 \\ Email: vdubey@bmth.ac.uk
}

\author{
Richard M Crowder \\ University of Southampton \\ Intelligence Agents Multimedia Group \\ Department of Electronics and Computer Science \\ Southampton, SO17 1BJ, UK \\ Tel. +442380593441 \\ Fax. +442380592865 \\ Email: rmc@ecs.soton.ac.uk
}

\begin{abstract}
This paper presents a design for a reconfigurable packaging system that can handle cartons of different shape and sizes and is amenable to ever changing demands of packaging industries for perfumery and cosmetic products. The system takes structure of a multi-fingered robot hand, which can provide fine motions, and dexterous manipulation capability that may be required in a typical packaging-assembly line. The paper outlines advanced modeling and simulation undertaken to design the packaging system and discusses the experimental work carried out. The new packaging system is based on the principle of reconfigurability, that shows adaptability to simple as well as complex carton geometry. The rationale of developing such a system is presented with description of its human equivalent. The hardware and software implementations are also discussed together with directions for future research.
\end{abstract}

Keywords: Packaging Machine, Carton Folding, Multifingered Hand, Flexible Automation, Mechanism Synthesis

\section{INTRODUCTION}

Product packaging is one of the key industrial sectors where automation is of paramount interest. The existing automation in packaging is however achieved by dedicated machines. These machines can handle only a fixed type of cartons, any change in shape and structure cannot be easily incorporated into the system. They require, in most cases, a change of more than 40 points [1] to fit into the same type of carton of different dimensions, which means one specific type of cartons requires one packaging line. Capital expenditure keeps on mounting while considering the change-over [2] from one type to another type of carton assembly line. The flexibility has thus been lost due to the limitations and the associated cost in the change-over.

Manual lines are thus introduced as a complement to adapting to different types of carton with fewer problems in the change-over. They take about $10 \%$ of the work order and are called upon as assembly lines to take the order of promotional products. However, the problem still emanates from the manual lines with a large learning curve for both supervisors and operators, and with inconsistency and labor injuries mostly due to hand twisting motions. Further, the manual line is generally considered to be the seasonal force that a dedicated machine still has to be used on a year-run basis to save cost and time. To make the task more difficult, the carton designers pursue fantasy and originality in carton packaging to respond to a highly competitive market. The frequent change of style and 
types of carton and the small batches of production present a challenge to the carton assembly and packaging, and demand a reconfigurable machine to be designed.

With the new trend for agile manufacturing, it is necessary to look for such a machine for the packaging industries to fully speed-up the change-over process for different types of carton and to even change the concept of "change-over" to a programmable and reconfigurable system; a system which can allow a quick change based on robotic systems in both assembly and packaging.

Development of such agile and highly reconfigurable systems requires systematic analysis and synthesis of each components, i.e. cartons and their folding patterns, machine operating on them, and the complete assembly operation itself. One such approach [3] has been reported for folding cartons with fixtures. While this approach generates all folding sequences for a carton, the implemented work just handles a simple rectangular carton for which fixed automation is already in place. For cartons of complex geometry, however, synthesis of both the carton and the folding mechanism needs to be considered together to achieve flexible automation in the assembly line. Extensive study has been undertaken by the authors on the operation of a complex carton folding pattern and its sequence analysis, resorting to graph theory, screw theory, matrix theory and representing cartons as a spatial mechanism; thereof studying the mobility and configuration of the carton [4-11]. This paper presents a joint study of carton folding and the newly designed mechanical device in a simulation environment, which led to the development of an experimental system. The developed system is unique which has shown to fold cartons of complex designs, exhibiting adaptability to meet ever-changing seasonal demands of innovative gift packaging. The paper introduces the original design, discusses advanced modeling and simulation techniques that has been used to carry out the experimental works.

\section{DESIGN PHILOSOPHY AND PRINCIPLES}

Study of manual packaging process reveals that a higher degree of dexterity is required as we move from cartons of simple types to a complex one [1]. Such cartons are formed on a pre-broken cardboard sheet of different geometrical shapes. As the sheet is broken, it has a number of movable panels which can be rotated about the lines of crease. These lines of creases are sometimes also referred to as 'crease lines', which facilitate the folding thus resulting in a shape transformation.

Figure 1 shows an example of a fancy carton, which after folding takes the final shape of the 'boy-scout-tent'; generally manual processes are resorted due to the complexity and typically small batch run of such cartons.

In manual process of packaging the side panels are bent using fingers since it requires folding along three axes as shown by the arrows in Fig. 2, while the top and bottom panels are bent using the palms, shown by the flat jaws. The arrows representing the fingers apply pointed force called 'poking' to the panels while the flat jaws apply 'pushing' force.

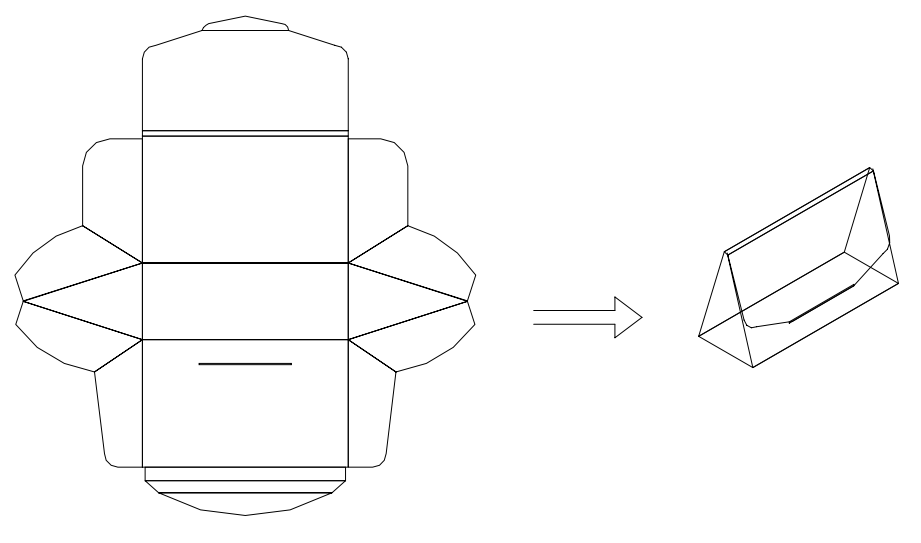

Fig. 1. Study of cardboard packaging

At intermediate level of closing, fine motions are required by the fingers to cross-lock the panels by inserting the projected panels into slit areas called the 'tucking' operation. These operations are further discussed in their context.

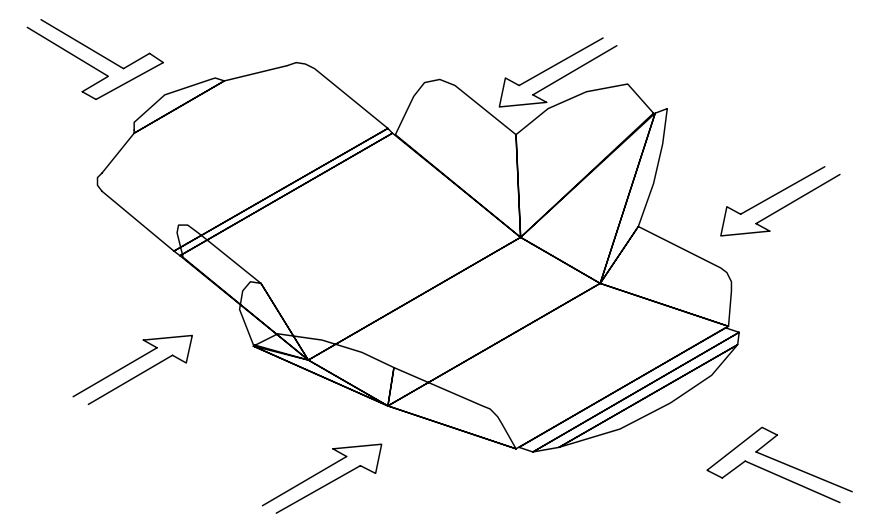

Fig. 2. Manipulative forces in manual packaging

In order to generalize the folding process, study of various cartons geometries are conducted that led to the following classification as shown in Fig. 3, it also shows various operations involved in cardboard packaging. It is important to note that most cartons have to pass through the three stations of erection, insertion and closure, however, except for the traytype cartons, the closing stations in other cases involve various manipulative functions (some of which are indicated above), depending on the complexity of the carton geometry.

For designing a mechanical system to erect and fold such cartons the following points need to be considered.

- Multiple functions: to provide various manipulative functions including poking, tucking, squeezing and twisting operations. 
- Dexterity: to reach the manipulative positions in different ways.

- Minimum number of axes of control: to reduce the complexity of the system.

- Reconfigurability: to handle variety of foldable cartons with different geometrical configurations.

- Programmability: to control motions on many axes simultaneously and sequentially.

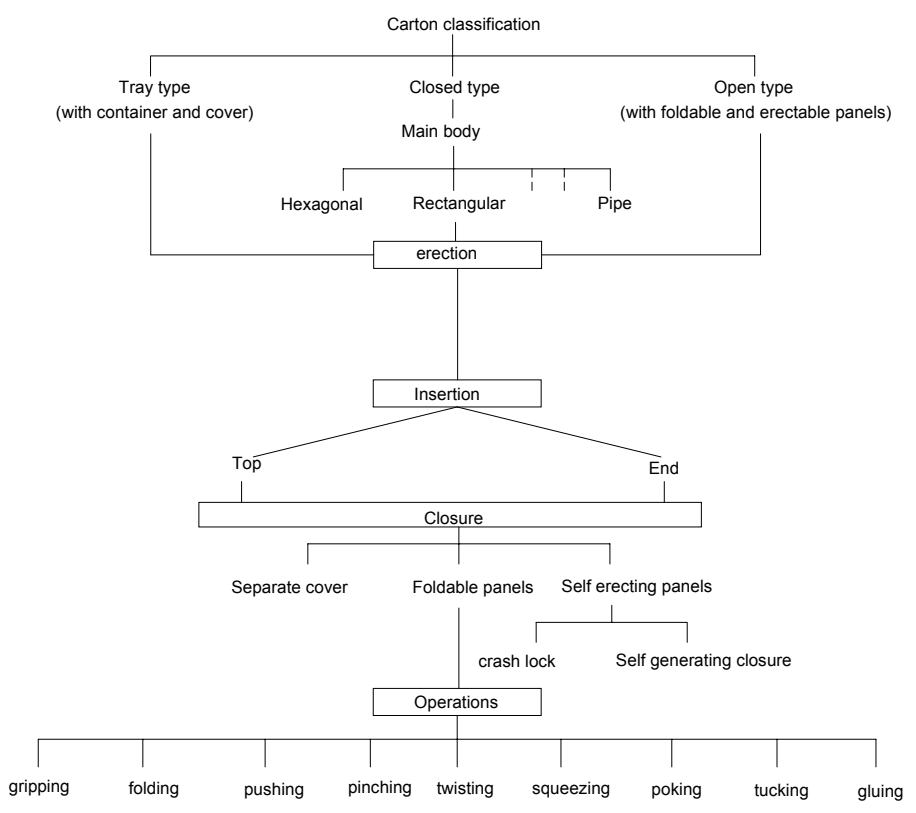

Fig. 3. Operations in carton packaging

A flexible system to provide fine motions and manipulative functions requires that articulated finger-like links be used. The reconfigurability of the system to handle cartons of different shapes and sizes can be ensured by mounting such fingers on a movable base, such as XY-table or circular tracks. The electronic architecture of the controller should be capable of moving each axis independently. The design should provide all manipulative functions to the fingers without making the system complex, thus resulting in a cost effective solution.

\section{THE PACKAGING MACHINE}

The conceptual design based on the above criterion is shown in the Fig. 4. The design has four fingers, two with three degrees of freedom, and two with two degrees of freedom each. The three-degree-of-freedom fingers provide a yaw motion at the base and pitch motions on the following two joints forming a Y-P-P configuration. The two-degree-of-freedom fingers have only pitch motions allowing it to move on a planar surface. These fingers are mounted on linear tracks that can move orthogonally and can be oriented at any angle at their base. Two horizontal jaws shown in the figure are arranged with the pushing direction parallel to the fingers' horizontal tracks. The plates attached to the jaws and are imagined to be mounted on passive joints that are under-actuated i.e. during the pushing operation, they can orient along the shape of the cardboard panels. The base of the system, on which the carton is attached, has a circular turntable that can rotate as well as move vertically up and down, thus allowing any orientation and elevation of carton to be achieved that may be required during packaging operation. These considerations were specifically based on providing high degree of reconfigurability with minimum number of axis to be controlled.

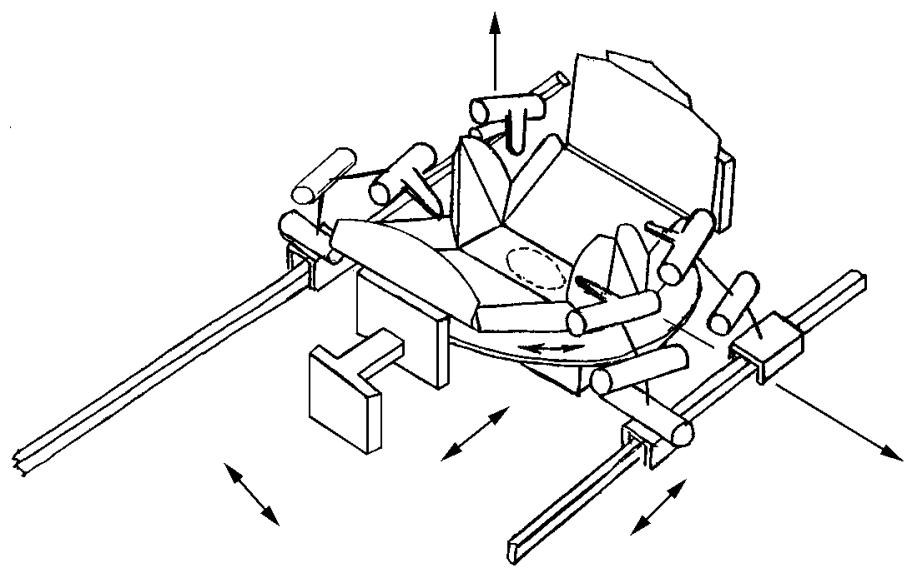

Fig. 4. Conceptual design of packaging machine

Based on the conceptual design a model was developed in a robotic simulation environment [12] with fingers and horizontal pushers as shown in Fig. 5. The base of the small vertical table at the center, which will hold the carton is shown to be actuated by a single motor for vertical movement as well as for the rotation of the table, however, in actual system these two axes will be controlled independently. As far as simulation is concerned, this model will provide all the kinematic information that may be required to run the machine. The fingers are seen to be mounted on tracks, which can slide along the track and the track itself can move laterally. Further the fingers are mounted on swivel-base that allows these axes to be oriented suitably. The joints of the fingers are actuated directly by joint-motors and the whole system has 14 controlled axes.

Special consideration has been given to design the fingertips, since they are required to perform various manipulative functions as discussed earlier. Inspired by the manual packaging processes, fingertip design incorporates pointed tip with V-groove to apply 'poking' and 'squeezing' effort to the cardboard panels as required in tucking operation. The pointed tip is used for poking operation while the V-groove holds the common crease line between the panels for squeezing to allow the panels to open up for tucking operation. The Y- 
shape of the two-degrees-of-freedom finger is to provide occasional pushing force on flat panels in addition to the poking and squeezing forces. Thus the design can provide the flexibility of offering many manipulative functions with limited degree of freedom to handle different types of cartons in different configurations.

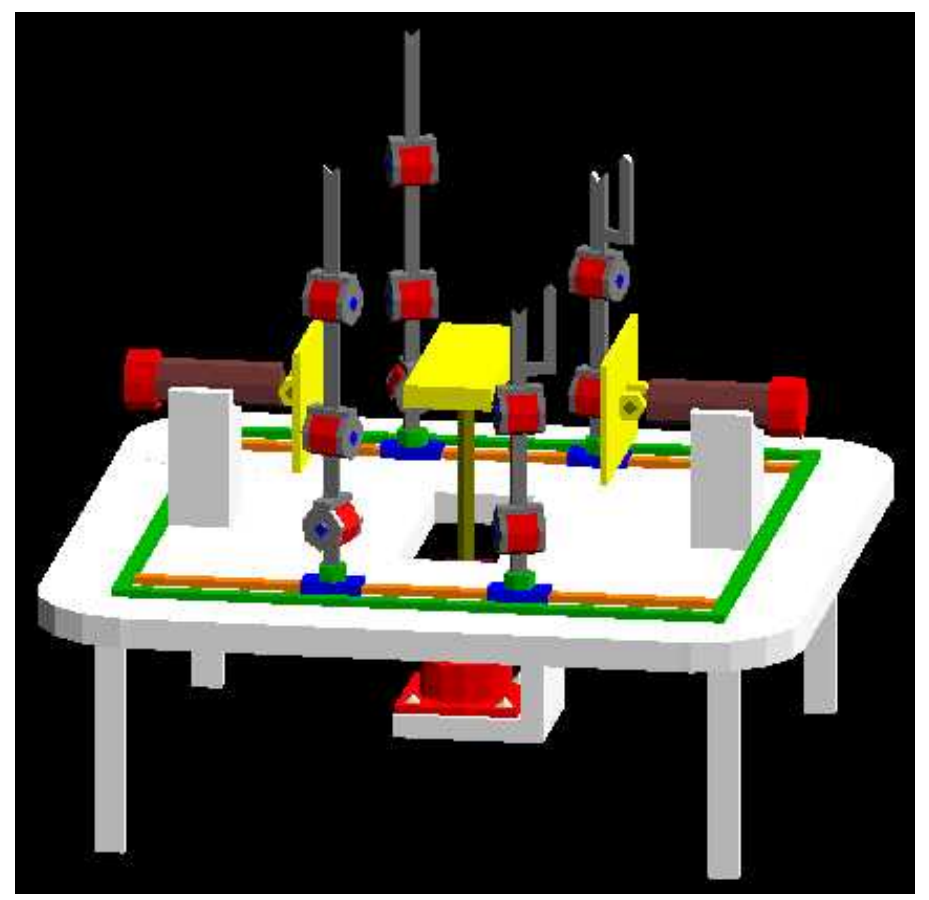

Fig. 5. Model of the packaging machine

The parametric modeling capability of the package allows geometrical and dimensional alterations in the design to be easily incorporated including the configuration verification. This also allows kinematic parameters of the machinecomponents to be ascertained before going in for the actual machine fabrication, this is discussed in the following section.

\section{SIMULATION OF PACKAGING PROCESS}

In order to simulate the packaging processes, kinematic model of carton as well as packaging machine components (fingers, pushers and the turn-table) need to be developed and integrated together. The kinematic model of the carton has been studied $[7,11]$, which is governed by two basic angles $(\beta$ and $\gamma)$ as shown in the Fig. 6 , and the corresponding equations result.

$$
\begin{aligned}
& \beta=\cos ^{-1}\left(\frac{R_{1} C \phi-R_{3} S \phi}{C \alpha}\right), \\
& \gamma=\cos ^{-1}\left(\frac{\left(R_{1} S \phi+R_{3} C \phi\right) C \alpha S \beta+R_{2} S \alpha}{S^{2} \alpha+(C \alpha S \beta)^{2}}\right)
\end{aligned}
$$

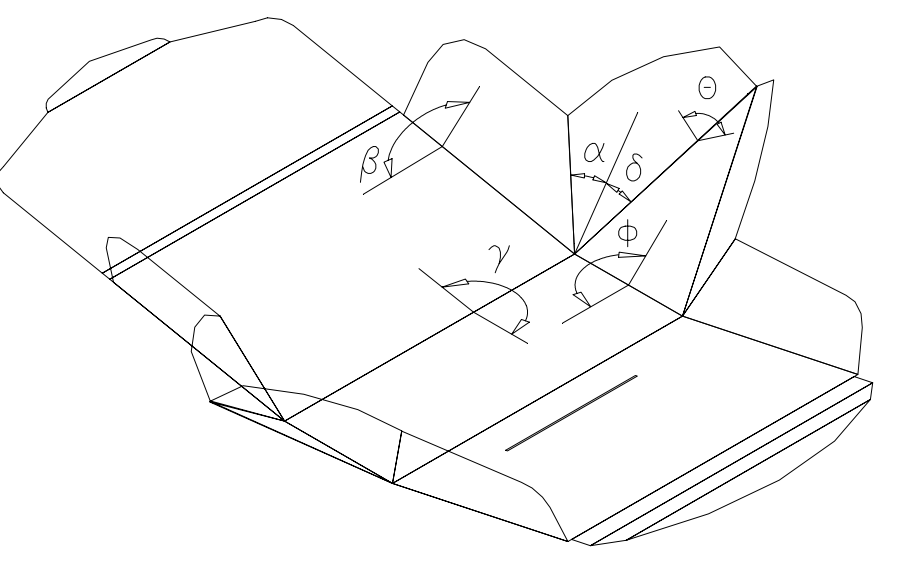

Fig. 6. Kinematic model of the carton

where,

$$
\begin{aligned}
& R_{1}=\left(C^{2} \delta V \theta+C \theta\right) C \alpha-C \delta S \delta V \theta S \alpha, \\
& R_{2}=\left(S^{2} \delta V \theta+C \theta\right) S \alpha-C \delta S \delta V \theta C \alpha, \\
& R_{3}=S \delta S \theta C \alpha+C \delta S \theta S \alpha, \\
& V \theta=(1-C \theta), S=\sin , C=\cos .
\end{aligned}
$$

These equations completely defines the folding sequence of the panels for proper shape transformation. If the folding of the carton is to be achieved by the packaging machine, each movable element of the machine needs to be connected kinematically. This requires inverse kinematic solution of the fingers to be developed. The 2-dof planar finger has standard solutions available [13], but solution for the 3-dof fingers with Y-P-P configuration is not very common, closed form solution for which is given by the following equations, which need to be implemented into the controller for tracking the carton-panel movement.

$$
\begin{aligned}
& \theta_{1}=\tan ^{-1}\left(\frac{p_{y}}{p_{x}}\right) \\
& \theta_{3}=\cos ^{-1}\left(\frac{p_{x}^{2}+p_{y}^{2}+p_{z}^{2}+l_{1}^{2}+2 l_{1}\left(p_{x} c_{1}+p_{y} s_{1}\right)-l_{2}^{2}-l_{3}^{2}}{2 l_{2} l_{3}}\right) \\
& \theta_{2}=\sin ^{-1}\left(\frac{\left(c_{1} l_{2}+l_{3} c_{1} c_{3}\right) p_{z}-\left(p_{x}-l_{1} c_{1}\right) s_{3} l_{3}}{\left(l_{2}+l_{3} c_{3}\right)\left(c_{1} l_{2}+l_{3} c_{1} c_{3}\right)+l_{3}^{2} c_{1} s_{3}^{2}}\right)
\end{aligned}
$$

where $p_{x}, p_{y}, p_{z}$ are the co-ordinates of the target point, $c$ and $s$ are sine and cosine of the angle, $l_{1}, l_{2}, l_{3}$ are the link lengths of the finger corresponding to joint angles $\theta_{1}, \theta_{2}$ and $\theta_{3}$ moving from base to the tip.

The kinematic connectivity between carton and the fingers is achieved by locating various contact points on the carton and recording the displacement of these points as the folding of the 
carton takes place. The contact points on the carton can be identified by geometrical interpretation of the folding sequences [11]. These contact points are then used to find joint displacement for each fingers' joints. The displacement data are further interpolated to generate optimal finger paths minimizing the unwanted fingers motion and thus reducing the packaging cycle-time. The interpolated data from the simulation can be downloaded to drive the actual fingers. This whole packaging process can also be automated based on the study of the geometric features of the panels and their folding sequences without resorting to the simulation of the carton, this is the current research effort [14].

Figure 7 shows the fingers tracking the contact points over the carton while it is folding. The simulation provides many valuable information that is used for experimental packagingmachine design as well as its control. For example, the simulation can be used for geometric as well as configuration checks before deciding on the dimension and structure of the machine. Any new geometrical information of the machine components can be directly obtained from the model by just making parametric changes in basic dimensions of the model. Motion-data and trajectory of the fingers so obtained during folding of the carton panels can be used for finger control in the actual system. Currently the motion parameter available from the simulation has not been directly integrated to the actual controller thus the data has to be fed off-line in the form of a data-file, which is read by the controller. Nevertheless, this technique allows full validation of folding sequence and then downloading these parameters to the controller.

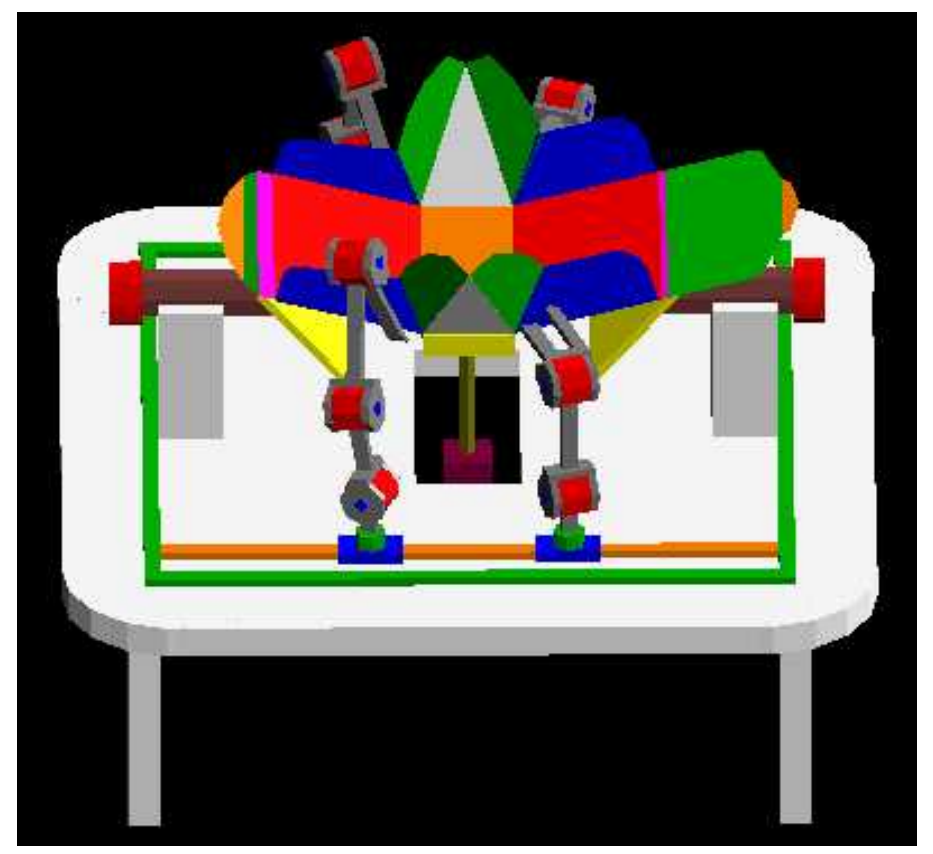

Fig. 7. Fingers tracking the carton folding

\section{THE EXPERIMENTAL MACHINE}

Using the dimensional information from the simulation, the experimental packaging machine was developed which uses three linear motors, two for jaw-pushers and one for the vertical motion of the turn-table. Ten high torque, high performance motors were used on the finger joints, specifications of the motors were:

Dimension: $\Phi 30 \times 30 \mathrm{~mm}$

Weight: 70 grams

Torque: $0.7 \mathrm{Nm}$ at $22.5 \mathrm{rpm}$

Gear ratio: 80:1, harmonic drive

Optical encoder with 96 pulse/rev.

This means $10 \mathrm{~cm}$ long arm connected to the motor can apply a fingertip force of $7 \mathrm{~N}$, which is high enough to fold the cardboard panels along the crease lines. The controller architecture of the system is shown in Fig. 8. It has four motion control cards and each can control up to 4 axes. These cards are supported by motion control library in C-programming language, it also has a G-code programming interface for quick trial and running motors in teach mode.

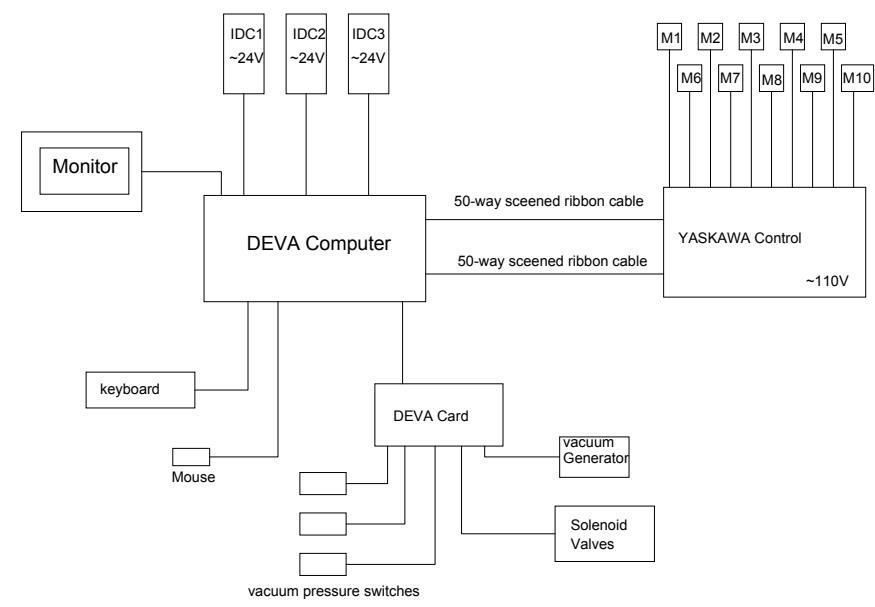

Fig. 8. The controller structure

The system also incorporates pneumatic connections for attaching suction cups, which can be activated in ON/OFF position by the controller. Currently this is employed to move the small turn-table from one orientation to the other and to hold the carton in fixed positions, however, at later stage it is planned to use these cups on the fingertips as well, for astrictive mode of grasping. This will have an advantageous effect in handling flat carton panels without slip [8].

In order to establish the capability of the packaging system in performing erection and folding of cartons, the data-file generated from the simulation was fed to the controller after ensuring that the both model and the machine have geometric and configuration equivalence. The data-file contains the 
motion data in a single row for simultaneous operation of the motors, whereas the succeeding lines have next stage motion control parameters. Accordingly, the controlling program reads the data file sequentially and generates appropriate interrupts within, to pass the motion commands simultaneously. Thus the fingers can duplicate motion in parallel as well as sequential modes. The programming capability of the controller can be further enhanced by developing sub-routines specific to various manipulative functions, thus allowing modular structure of the controller to be achieved, which can be easily adapted to any new carton folding and packaging sequences.

\section{EXPERIMENTATION}

Reconfigurability of the system was one of the key issues for which this system was developed. The idea was to use the system to fold different type of cartons with little alteration in the system design. But to achieve this, it is important to ensure that the system is exactly configured as per the developed graphical model and the basic structural set up. The data file is included in the main program and initially the system is run in step-mode to verify the fingers' movement while the carton is folding, once this is done the system is ready for automated packaging. The developed machine is shown in Fig. 9 where all fingers and horizontal pushers are involved in a complicated sequence of tucking operation. The system was able to erect and fold the carton successfully in less than 45 seconds.

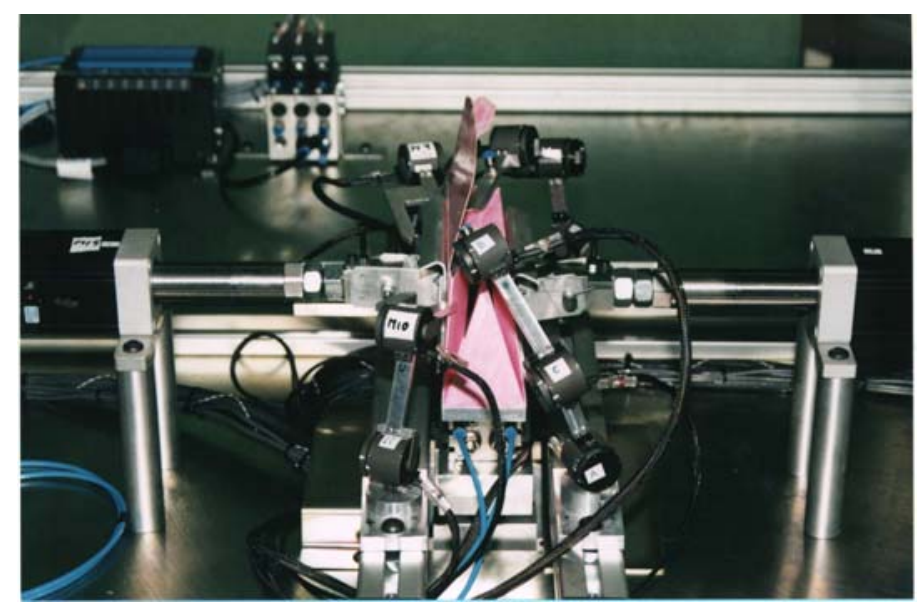

Fig. 9. The experimental packaging machine

Reconfigurability of the machine was demonstrated by folding a second type of carton as shown in Fig. 10. This carton is a closed-type of completely different shape and complexity with multiple flaps. This requires various manipulative functions of pocking, twisting and tucking to be performed on the panels. After reconfiguring the structure of the machine, the folding sequences took a cycle time of 45 seconds to complete the carton. The reconfigured machine can be compared with the previous set-up in Fig. 9. This demonstrates the highly flexible and reconfigurable design of the system.

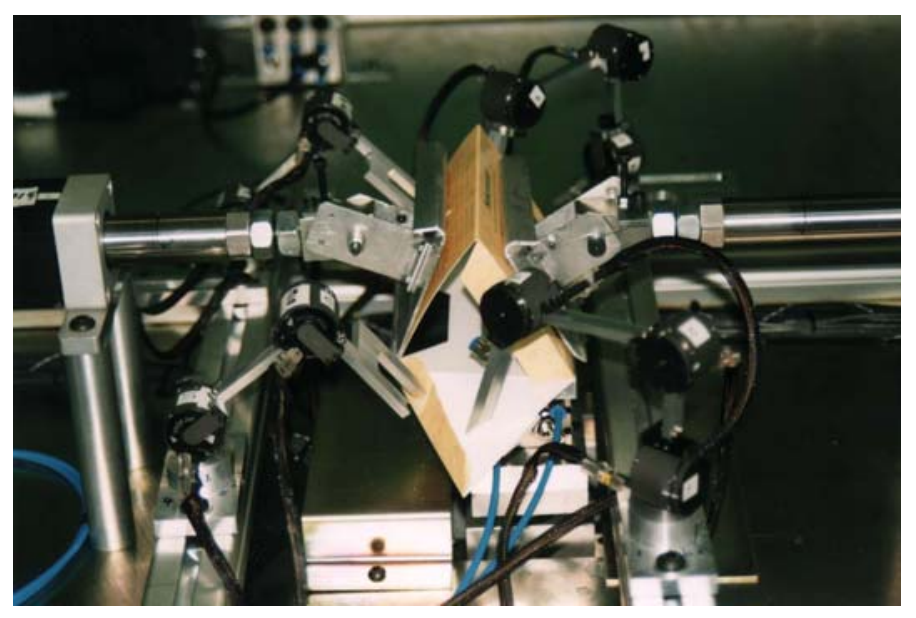

Fig. 10. Fingers twisting the carton panel

\section{DISCUSSIONS AND CONCLUSIONS}

This paper has presented a dexterous reconfigurable assembly and packaging system. The aim of this work was to design a reconfigurable assembly and packaging system that can handle cardboard cartons of different shapes and sizes. The initial idea was to develop such a system that can demonstrate adaptability to cartons of different styles and complexities. It was shown that the packaging machine could fold two cartons of completely different shapes. The cycle time for the folding was approximately 45 seconds in each case. Though this is not the optimized time of folding, it is hoped to reduce the cycle time to 30 seconds or less with on-line data transfer. Although there are many issues that need to be addressed before a full flexible machine can be realized, nevertheless, the research was aimed at proving the technology for quick change-over, which faces the industry in the new trends of agile manufacturing.

The future enhancement will include optimization of finger trajectory, use of tactile sensors for force feedback to avoid excessive pressure on the panel, and astrictive mode of grasping to involve the vacuum system at the fingertips. It is also proposed to integrate the simulation model directly with the actual machine to download the motion control data online. The XY-table can be motorized and controlled for autoreconfiguration. These advanced techniques will automate the entire packaging process starting from the two dimensional drawing of the cardboard, defining its kinematic model then generating the motion sequences leading to the finished product package. It is also envisaged to mount such dexterous reconfigurable hands onto a robotic arm to offer higher level of flexibility in packaging. The system will not only perform carton folding but can also be used to insert the product into the carton during the folding sequences. This will reduce the packaging time and will also be able to meet challenges of high adaptability to ever changing demands of high-end personal 
product packaging. The new design has shown a strategy of folding complex cartons that has so far been realized as the bottleneck to the perfumery and cosmetic industries, thus providing a step forward to the concept of flexible automation for the product packaging and assembly lines.

\section{ACKNOWLEDGMENTS}

This research was supported by a grant from the Unilever Research, Port Sunlight, Wirral (UK), which the authors highly appreciate. The authors also acknowledge the initial contributions of previous researchers, in particular, Jian Dai for laying the foundation for this research.

\section{REFERENCES}

[1] Dai, J. S., 1996, "Survey and Business Case Study of the Dextrous Reconfigurable Assembly and Packaging System (DRAPS)", Science and Technology Report, No PS960321, Unilever Research (UK).

[2] Dai, J. S., 1996, "Conceptual Design of the Dextrous Reconfigurable Assembly and Packaging System (D-RAPS)", Science and Technology Report, No PS960326, Unilever Research (UK).

[3] Lu, L and Akella, S., 2000, "Folding cartons with fixtures: a motion planning approach" IEEE Transactions on Robotics and Automation, Vol. 16, No. 4, pp. 346-356.

[4] Dai, J. and Rees Jones, J., 1997, "New Models for identification of distinct configurations of Cartons during Erection and Assembly", Science and Technology Report, PS 97 0066, Unilever Research (UK).

[5] Dai, J. S. and Rees Jones, J., 1997, "Structure and Mobility of Cartons through Screw Theory", Science and Technology Report, PS 97 0067, Unilever Research (UK).
[6] Dubey, V. N., Dai, J. S. and Stamp, K. J., 1999, “Advanced Modeling and Kinematic Simulation of a Complex Carton" Science and Technology Report, Unilever Research, (UK).

[7] Dubey, V.N., Dai, J. S. Stamp, K. J. and Rees Jones, J., 1999, "Kinematic Simulation of a Metamorphic Mechanism", Tenth World Congress on the Theory of Machine and Mechanisms (IFToMM), pp. 98-103.

[8] Dubey, V. N., Dai, J. S. and Stamp, K. J., 1999, “Advanced Modelling, Design and Experimental Work of a New Reconfigurable System for Packaging with a Mult-fingered Robot Hand", Science and Technology Report, Unilever Research, (UK).

[9] Dai, J. S. and Rees Jones, J., 1997, "Theory on Kinematic Synthesis and Motion Analysis of Cartons", Science and Technology Report, PS 97 0184, Unilever Research (UK).

[10] Dai, J. S. and Rees Jones, J., Mobility in Metamorphic Mechanisms of Foldable/Erectable Kinds, ASME Journal of Mechanical Design, Vol. 21, No. 3, 1999, pp. 375-382.

[11] Dubey, V.N. and Dai J. S., 2001, "Modeling and Kinematic Simulation of a Mechanism extracted from a Cardboard Fold", International Journal of Engineering Simulation, Vol. 2, No. 3, pp. 3-10.

[12] Workspace4, 1998, User's Guide, Robot Simulations Ltd., UK.

[13] Fu, K. S., Gonzalez, R. C. and Lee C. S. G., 1987, Robotics: Control, Sensing, Vision, and Intelligence, McGrawHill International, New York.

[14] Dubey, V.N., Dai, J. S. and Stamp, K. J., 2000, "Knowledge-based Carton Specification System" Science \& Technology Report, Unilever Research, (UK). 